\title{
GMI Effect of Ultra-Soft Magnetic Soft Amorphous Microwires
}

\author{
A. Zhukov ${ }^{*}{ }^{, 1,2}$, M. Ipatov ${ }^{2}$, J.M. Blanco ${ }^{3}$ and V. Zhukova ${ }^{2}$ \\ ${ }^{1}$ IKERBASQUE, Basque Foundation for Science, 48011 Bilbao, Spain \\ ${ }^{2}$ Dpto. Fisica de Materiales, Fac. Quimicas, UPV/EHU, 20009 San Sebastian, Spain \\ ${ }^{3}$ Dpto. de Física Aplicada, EUPDS, UPV/EHU, 20018, San Sebastian, Spain
}

\begin{abstract}
In this paper we experimentally studied GMI effect and soft magnetic behavior of Co-rich microwires. Correlation between magnetoelastic anisotropy and magnetic field dependences of diagonal and off-diagonal impedance components are observed. Low field GMI hysteresis, explained in terms of magnetoelastic anisotropy of microwires, has been suppressed by the bias current.
\end{abstract}

Keywords: Amorphous wires and microwires, giant magneto-impedance, skin effect, magnetic anisotropy.

\section{INTRODUCTION}

Magnetically soft glass coated microwires (typically of 5$30 \mu \mathrm{m}$ in diameter) exhibit a number of outstanding magnetic properties such as magnetic bistability and giant magneto-impedance, GMI, effect [1,2]. Recent studies allowed improvement of magnetically soft properties and GMI effect of glass coated microwires [2, 3]. It must be mentioned, that recently fabrication of extra-thin glasscoated microwires with metallic nucleus diameter as low as $100 \mathrm{~nm}$ is recently reported, although GMI effect is studied in samples with diameters above $3 \mu \mathrm{m}[2,4]$.

Giant magneto-impedance effect (GMI), consisting of large sensitivity of the impedance of magnetically soft conductor on applied magnetic field, attracted great attention in the field of applied magnetism [2-7] especially because of excellent magnetic field sensitivity suitable for low magnetic field detection. Such GMI effect is especially high in ferromagnetic magnetically soft wires (especially of amorphous and nanocrystalline origin) [2, 6, 7]. It is worth mentioning, that the cylindrical shape is quite suitable for achieving of high GMI effect [2, 6,7]. General tendency on miniaturization of magnetic sensors requires development of thin soft magnetic materials, like thin wires and thin films. Owing to its thin dimensions and outstanding magnetic properties such as magnetic bistability and GMI effect, glasscoated microwires gained special interest in the field of applied magnetism for designing of the sensors based on GMI effect [2, 8]. Recently, excellent soft magnetic properties and GMI effect of glass coated microwires attracted great attention $[3,5]$, giving rise to development of industrial applications for low magnetic field detection $[9,10]$. Most recent applications derive from the GMI effect in the $\mathrm{MHz}$ frequency range observed in non-magnetostrictive microwires. Co- based microwires are currently employed by

*Address correspondence to this author at the IKERBASQUE, Basque Foundation for Science, and Dpto. Fisica de Materiales, Fac. Quimicas, UPV/EHU, 20009 San Sebastian, Spain; Tel: 34-943018611;

Fax: 34-943017130; E-mails: arkadi.joukov@ehu.es, arcadyzh@mail.ru
Aichi Steels in Japan integrated in CMOS circuit as kind of magnetic compass for a number of uses in mobile systems [9]. A magnetic sensor making use of that effect developed by $\mathrm{K}$. Mohri and T. Uchiyama is characterized by quite elevated sensitivity of $1 \mathrm{pT}[10]$.

In most of applications a high linearity of MI dependence and low hysteresis are desirable [2, 10]. Anti-symmetrical MI curve with linear region and low noise features have been obtained in current pulsed excitation scheme of wires using detection of off-diagonal GMI component $[2,10,11]$. At the same time we have recently showed, that linearity and shape of off-diagonal component in microwires can be tailored by thermal treatment $[2,13,14]$. Considerable GMI hysteresis has been observed and analyzed in microwires possessing helical anisotropy [10], although enhanced magnetic field sensitivity of the GMI effect in amorphous wires is related to specific outer domain structure in the surface area [13-17].

In this paper we studied the GMI effect (GMI ratio, $\Delta \mathrm{Z} / \mathrm{Z}$, diagonal $Z_{z z}$ and off-diagonal impedance tensor $Z_{\phi z}$ components) and hysteretic magnetic properties in ultra-thin amorphous glass-coated microwires with vanishing magnetostriction constant.

\section{EXPERIMENTAL DETAILS}

We have measured dependences of the diagonal $Z_{z z}$ and off-diagonal $Z_{\varphi_{z}}$ impedance components on external axial magnetic field $H$ in Co-rich microwires, as described elsewhere $[2,13-16]$. The microwires with nominal composition.

$\mathrm{Co}_{67.1} \mathrm{Fe}_{3.8} \mathrm{Ni}_{1.4} \mathrm{Si}_{14.5} \mathrm{~B}_{11.5} \mathrm{Mo}_{1.7}$ and $\mathrm{Co}_{66} \mathrm{Cr}_{3.5} \mathrm{Fe}_{3.5} \mathrm{~B}_{16} \mathrm{Si}_{11}$ different diameters of metallic nucleus, $\mathrm{d}$, and total diameters, $\mathrm{D}$, have been fabricated by the Taylor-Ulitovsky method, as described elsewhere [2]. Studied microwires have negtive vanishing magnetostriction constant and diameters of metallic nucleus from 6 to $22 \mu \mathrm{m}$. It is worth mentioning, that the magnetostriction constant, $\lambda_{\mathrm{s}}$, in system $\left(\mathrm{Co}_{\mathrm{x}} \mathrm{Fe}_{1}\right.$ $\left.{ }_{x}\right)_{75} \mathrm{Si}_{15} \mathrm{~B}_{10}$ changes with $\mathrm{x}$ from $-5 \times 10^{-6}$ at $\mathrm{x}=1$, to $\lambda_{\mathrm{s}} \approx 35 \times 10^{-6}$ at $x \approx 0.2$ [2]. Therefore, producing microwires we selected the $\mathrm{Fe}$ and $\mathrm{Co}$ content for obtaining vanishing 
magnetostriction constant in order to achieve better soft magnetic properties.

The microwires were placed in a specially designed microstrip cell. One wire end was connected to the inner conductor of a coaxial line through a matched microstrip line while the other was connected to the ground plane. The components $Z_{z z}$ and $Z_{\varphi_{z}}$ were measured simultaneously using vector network analyzer. The diagonal impedance of the sample $Z w=Z z z l$, where $l$ is the wire length, was obtained from reflection coefficient $S_{11}$ and the off-diagonal impedance $Z_{\varphi_{z}}$ was measured as transmission coefficient $S_{21}$ as a voltage induced in a $2-\mathrm{mm}$ long pick-up coil wounded over the wire. The static bias field $H_{B}$ was created by the dc current $I_{B}$ applied to sample through the bias-tee element. The frequency range for the off-diagonal component $Z_{\varphi_{z}}$ was $10-300 \mathrm{MHz}$, while diagonal impedance component has been measured till $7 \mathrm{GHz}$. The sample holder was placed inside a sufficiently long solenoid that creates a homogeneous magnetic field, $H$. More details on experimental technique can be found in refs. [2, 11].

For practical sensor it is essential to have the antisymmetrical dependence on magnetic field, so pulsed GMI effect allowing detection of the off-diagonal MI components could be more suitable $[5,10,12]$. The other reason is that in the sensor application, pulse excitation is preferred over sinusoidal because of simple electronic design and low power consumption, therefore we also used pulsed excitation scheme, as described elsewhere [5, 10, 12].

Hysteresis loops have been measured by the induction method, as described elsewhere $[2,13]$.

\section{RESULTS AND DISCUSSION}

Magnetic field, $\mathrm{H}$, dependence of real part, $Z_{l}$ of the longitudinal wire impedance $Z_{\mathrm{zz}}\left(Z_{\mathrm{zz}}=\mathrm{Z}_{1}+\mathrm{i} \mathrm{Z}_{2}\right)$, measured till $4 \mathrm{GHz}$ in $\mathrm{Co}_{66} \mathrm{Cr}_{3.5} \mathrm{Fe}_{3.5} \mathrm{~B}_{16} \mathrm{Si}_{11}$ microwire is shown in Fig. (1). The general features of these dependences are that the magnetic field of maximum shifts to the higher field region with increasing the $f$. On the other hand, we observed GMI effect (change of impedance under application of magnetic field) even at $\mathrm{GHz}$ - range frequencies.

Off-diagonal and low field diagonal components of GMI, measured in $\mathrm{Co}_{67} \mathrm{Fe}_{3.85} \mathrm{Ni}_{1.45} \mathrm{~B}_{11.5} \mathrm{Si}_{14.5} \mathrm{Mo}_{1.7}$ microwire are shown in Fig. (2). Important feature of Fig. (2) is a considerable hysteresis for both off-diagonal and longitudinal impedance.

It is worth mentioning, that in pulsed exciting scheme when the sharp pulses with pulse edge time about $5 \mathrm{~ns}$ are produced by passing square wave multi-vibrator pulses through the differentiating circuit, overall pulsed current contains a DC component that produces bias circular magnetic field $[3,5,11,15,16]$. In this way low field hysteresis can be surpassed selecting adequate pulse amplitude.

On the other hand, pulsed wire excitation is quite suitable for the electronic circuits for designing the sensors. Such scheme using pulsed excitation of magnetically soft wires with GMI effect (pulsed GMI effect) generating antisymmetric output in the pick-up coil surrounding wire and originating from off-diagonal MI component has been recently proposed [12].
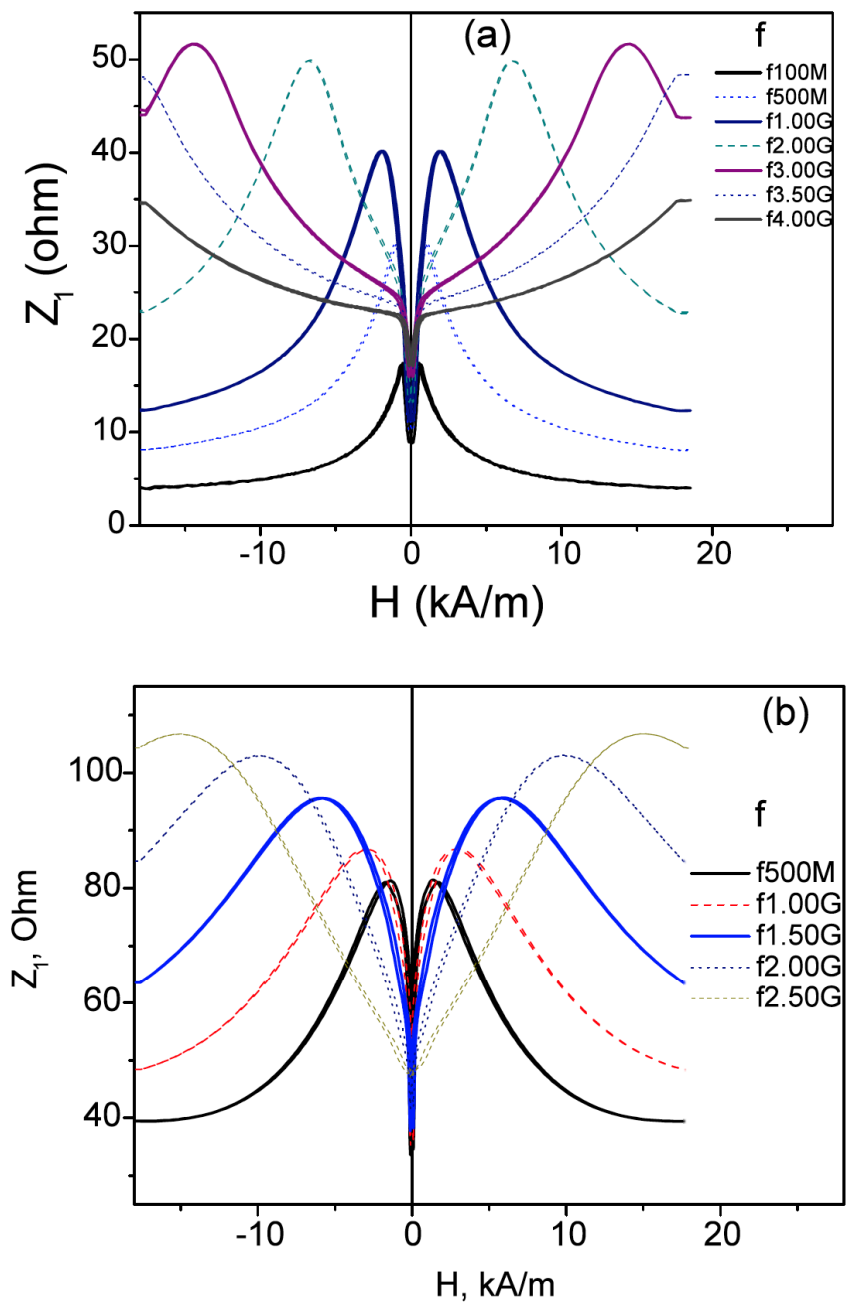

Fig. (1). $\mathrm{Z}(\mathrm{H})$ dependence of $\mathrm{Co}_{66} \mathrm{Cr}_{3.5} \mathrm{Fe}_{3.5} \mathrm{~B}_{16} \mathrm{Si}_{11}$ (a) and $\mathrm{Co}_{67} \mathrm{Fe}_{3.85} \mathrm{Ni}_{1.45} \mathrm{~B}_{11.5} \mathrm{Si}_{14.5} \mathrm{Mo}_{1.7}$ (b) microwires measured at different frequencies.

It is worth mentioning, that the preparation of glasscoated microwires involving simultaneous solidification of composite microwire consisting of ferromagnetic metallic nucleus inside the glass coating introduces considerable residual stresses inside the ferromagnetic metallic nucleus [2]. The strength of internal stresses is determined by thickness of glass coating and metallic nucleus diameter, i.e. by the $\rho$-ratio $(\rho=d / D)$.

Fig. (3) shows field dependence of the off-diagonal voltage response, $V_{\text {out }}$ measured using pulsed scheme, described elsewhere $[2,12]$ in $\mathrm{Co}_{67.1} \mathrm{Fe}_{3.8} \mathrm{Ni}_{1.4} \mathrm{Si}_{14.5} \mathrm{~B}_{11.5} \mathrm{Mo}_{1.7}$ $\left(\lambda_{s} \approx-3 \cdot 10^{-7}\right)$ microwire with different geometry: metallic nucleus diameter and total diameter with metallic nucleus diameters 6 and $7 \mu \mathrm{m}$ and $\rho$-ratios 0,59 and 0,64. The offdiagonal components exhibit anti-symmetrical magnetic field dependence, suitable for determination the magnetic field direction in real sensor devices [2, 10,12]. It should be noted from Fig. (3) that the $V_{\text {out }}(H)$ curves exhibit nearly linear growth within the field range from $-H_{m}$ to $H_{m}$. The $H_{m}$ limits the working range of MI sensor to $240 \mathrm{~A} / \mathrm{m}$ and should be associated with the anisotropy field. The effect of the $\rho$-ratio on $V_{\text {out }}(H)$ (Fig. 3) should be attributed to the 

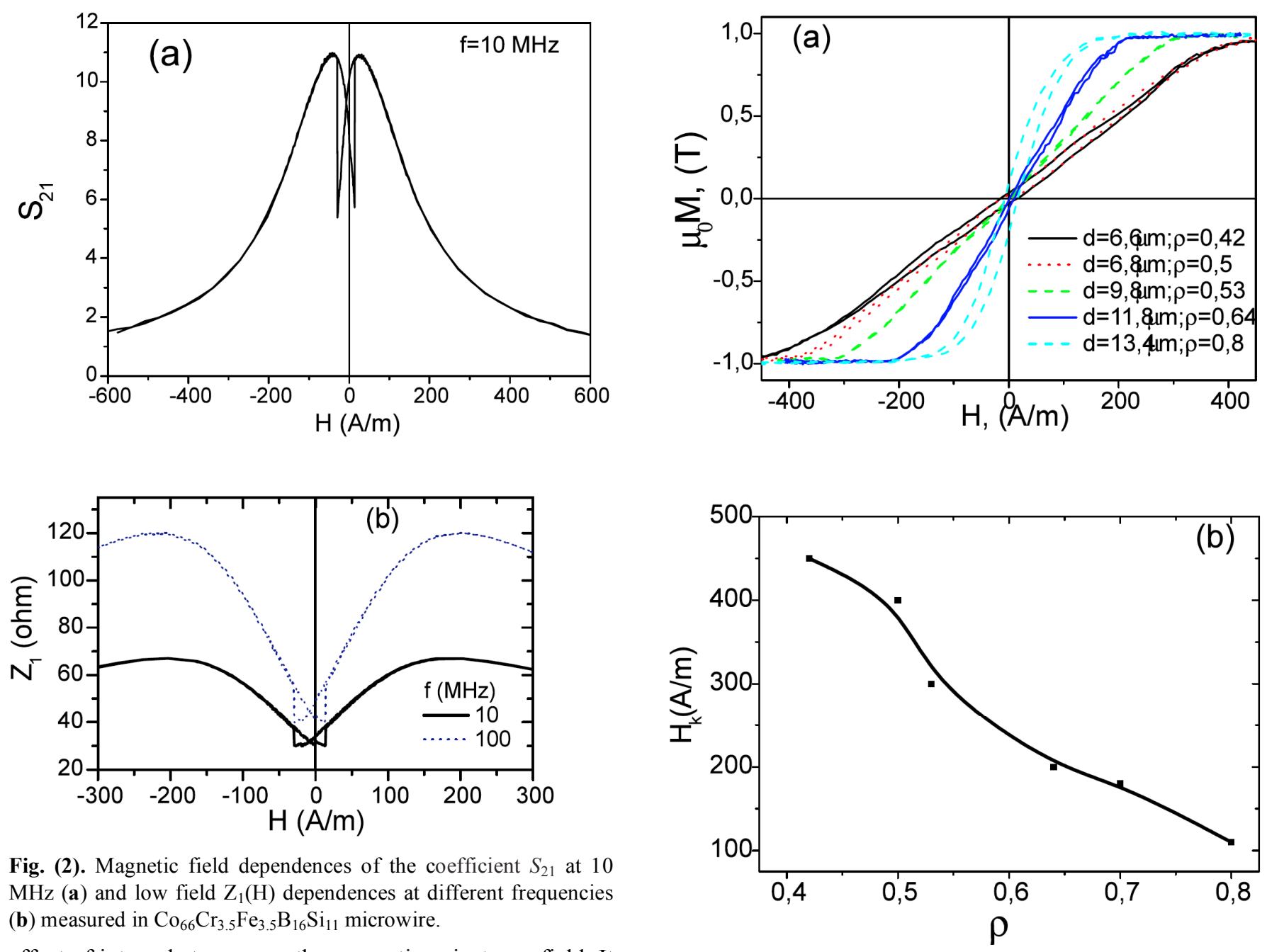

Fig. (2). Magnetic field dependences of the coefficient $S_{21}$ at 10 $\mathrm{MHz}$ (a) and low field $\mathrm{Z}_{1}(\mathrm{H})$ dependences at different frequencies (b) measured in $\mathrm{Co}_{66} \mathrm{Cr}_{3.5} \mathrm{Fe}_{3.5} \mathrm{~B}_{16} \mathrm{Si}_{11}$ microwire.

effect of internal stresses on the magnetic anisotropy field. It must be underlined, that all studied samples exhibited excellent magnetically soft properties with inclined hysteresis hoops and extremely low coercivities (between 4 and $10 \mathrm{~A} / \mathrm{m})$. Magnetic anisotropy field, $H_{k}$, is found to be determined by the $\rho$-ratio, decreasing with $\rho$ (Fig. 4), as also has been reported earlier $[2,13]$.

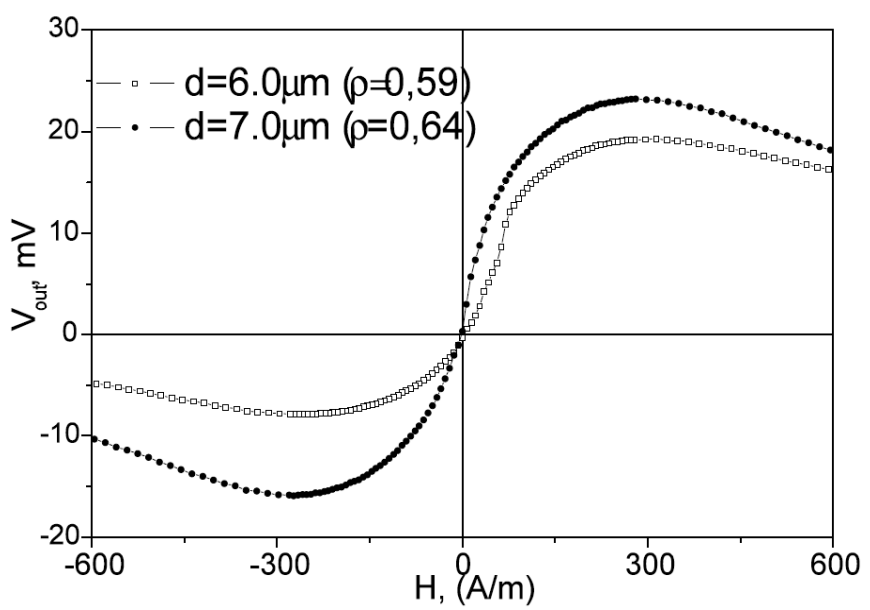

Fig. (3). $\mathrm{V}_{\text {out }}(\mathrm{H})$ response of $\mathrm{Co}_{67,1} \mathrm{Fe}_{3,8} \mathrm{Ni}_{1,4} \quad \mathrm{Si}_{14,5} \mathrm{~B}_{11,5} \mathrm{Mo}_{1,7}$ microwires with different diameters, $\mathrm{d}$.

Fig. (4). Hysteresis loops of $\mathrm{Co}_{67.1} \mathrm{Fe}_{3.8} \mathrm{Ni}_{1.4} \quad \mathrm{Si}_{14.5} \mathrm{~B}_{11.5} \quad \mathrm{Mo}_{1.7}$ microwires with different geometry (a) and dependence of $\mathrm{H}_{\mathrm{k}}$ on $\rho$-ratio (b).

On the other hand, since the strength of internal stresses, $\sigma_{i}$, arising during simultaneous rapid quenching of metallic nucleus surrounding by the glass coating can be controlled by the $\rho$-ratio, magnetic field dependences of both $Z_{z z}$ and $Z_{\varphi_{z}}$ can be manupulated by the $\rho$-ratio.

Additionally, we recently found, that the nature of observed low field hysteresis on $Z_{1}(H)$ and $Z_{\varphi_{z}}(\mathrm{H})$ is directly related with deviation of the anisotropy easy axis from transversal direction [11]. Therefore, application of circular bias magnetic field $H_{B}$ produced by DC current $I_{B}$ running through the wire affects the hysteresis and asymmetry of the MI dependence, suppressing this hysteresis when $I_{B}$ is high enough (see Fig. 5, where effect of bias voltage on diagonal impedance, $Z_{1}$, and on $S_{21}$ parameter. proportional to offdiagonal GMI component is shown). In fact in pulsed exciting scheme when the sharp pulses with pulse edge time about $5 \mathrm{~ns}$ are produced by passing square wave multivibrator pulses through the differentiating circuit, overall pulsed current contains a DC component that produces bias circular magnetic field [2,11]. In this way low field hysteresis can be surpassed selecting adequate pulse amplitude. 

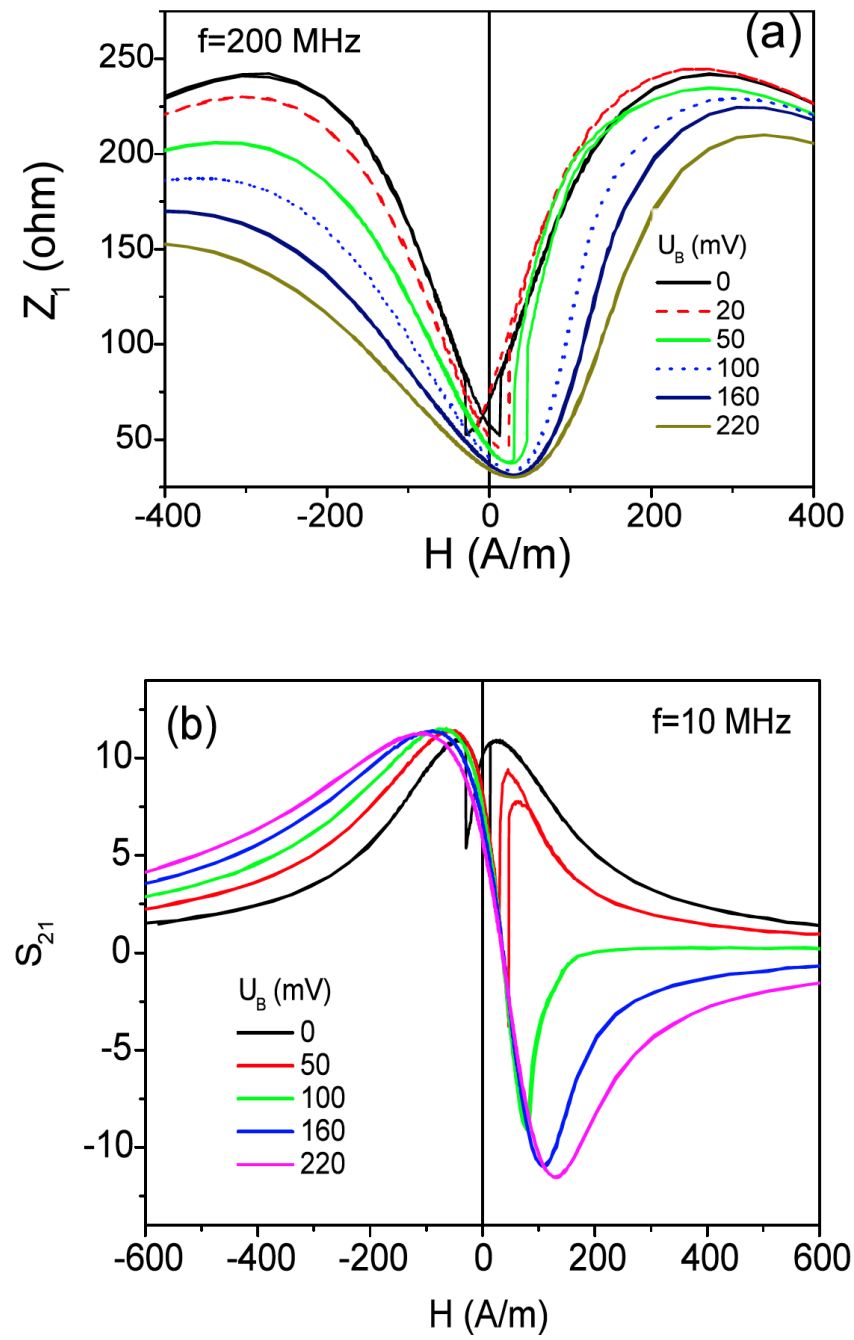

Fig. (5). Effect of bias voltage $U_{B}$ on magnetic field dependence of diagonal impedance (a) and $\mathrm{S}_{21}$ parameter (b) of $\mathrm{Co}_{67} \mathrm{Fe}_{3.85} \mathrm{Ni}_{1.45}$ $\mathrm{B}_{11.5} \mathrm{Si}_{14.5} \mathrm{Mo}_{1.7}$ microwire.

Traditional way to tailor magnetoelastic anisotropy is a thermal treatment [2]. We used Joule heating flowing the electrical current of $50 \mathrm{~mA}$ through the samples varying the duration from 1 to $20 \mathrm{~min}$. The influence of Joule heating on off-diagonal field characteristic of nearly zero magnetostriction $\mathrm{Co}_{67.1} \mathrm{Fe}_{3.8} \mathrm{Ni}_{1.4} \mathrm{Si}_{14.5} \mathrm{~B}_{11.5} \mathrm{Mo}_{1.7}$ microwire with $\rho=0,55(d=9.4, D=17.0 \mu \mathrm{m})$ is shown in Fig. (6). One can see that the thermal annealing with $50 \mathrm{~mA} \mathrm{DC}$ current reduces the $H_{m}$ from $480 \mathrm{~A} / \mathrm{m}$ in as-cast state to $240 \mathrm{~A} / \mathrm{m}$ after 5 min annealing.

Observed $H_{k}(\rho)$ dependence has been attributed to the magnetoelastic energy contribution given by

$K_{m e} \approx 3 / 2 \lambda_{s} \sigma_{v}$,

where $\lambda_{s}$ is the saturation magnetostriction and $\sigma_{i}$ is the internal stress. The magnetostriction constant is mostly determined by the chemical composition and achieves almost nearly-zero values in amorphous alloys based on $\mathrm{Fe}$ Co with $\mathrm{Co} / \mathrm{Fe} \approx 70 / 5 \lambda_{s} \approx 0[2,18]$. On the other hand, the estimated values of the internal stresses in these glass coated microwires arising from the difference in the thermal expansion coefficients of simultaneously solidifying metallic nucleus and glass coating are of the order of 100-1000 $\mathrm{MPa}$, depending strongly on the ratio between the glass coating thickness and metallic core diameter [2, 18, 19], increasing with decreasing $\rho$-ratio. Consequently, magnetoelastic anisotropy of glass-coated microwires can be controlled by the geometrical ratio $\rho$ through the strength of internal stresses.

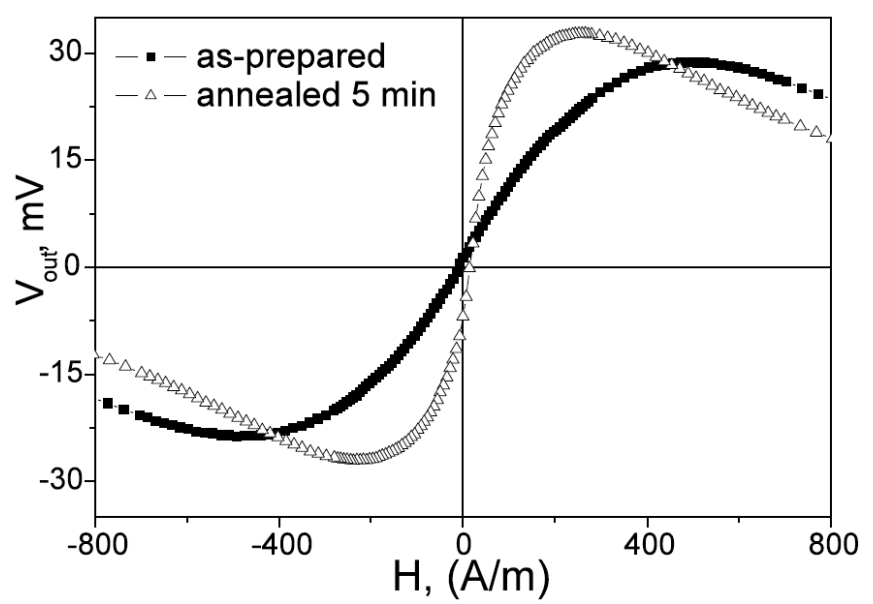

Fig. (6). $V_{\text {out }}(H)$ of as -prepared and Joule-heated $\mathrm{Co}_{67} \mathrm{Fe}_{3.85} \mathrm{Ni}_{1.45} \mathrm{~B}_{11.5} \mathrm{Si}_{14.5} \mathrm{Mo}_{1.7}$ microwire (current annealing with $50 \mathrm{~mA}$ current intensity).

Application of stress during stress annealing of Fe-rich microwires resulted in induction of considerable stress induced anisotropy [20]. In this case the easy axis of magnetic anisotropy has been changed from axial to transversal [19]. Origin of such stress-induced anisotropy is related with so-called "Back stresses" originated from the composite origin of glass-coated microwires annealed under tensile stress: compressive stresses compensate axial stress component and under these conditions transversal stress components are predominant [20].

Consequently, these stress annealed samples exhibit stress-impedance effect, i.e. impedance change $(\Delta Z / Z)$ under applied stress, $\sigma$, observed in samples with stress induced transversal anisotropy.

It should be assumed that the internal stresses relaxation after heat treatment should drastically change both the soft magnetic behavior and $\Delta Z / Z(H)$ dependence due to stress relaxation, induced magnetic anisotropy and change of magnetostriction constant under annealing.

\section{CONCLUSIONS}

In thin amorphous wires, produced by the TaylorUlitovski technique, magnetic softness and magnetic field dependence of GMI effect (both longitudinal and offdiagonal) and GMI hysteresis are determined by the magnetoelastic anisotropy. This magnetoelastic anisotropy can be tailored by the sample geometry and adequate annealing. There are a number of interesting effects, such as induction of the transversal anisotropy in Fe-rich microwires allowing creating extremely stress sensitive elements. Studies of diagonal and off-diagonal MI tensor components of glass-coated microwires have shown the great potential of these materials for microminiaturized magnetic field sensor application. Their main advantages are high sensitive lowhysteresis field dependence. By varying the alloys 
composition and applying post fabrication processing it is possible to control the sensor's operating range. Low field GMI hysteresis has been observed and explained in terms of helical magnetic anisotropy of microwires.

\section{ACKNOWLEDGMENT}

This work was supported by EU ERA-NET programme under "SoMaMicSens" (MANUNET-2010-Basque-3) project, by EU under FP7 "EM-safety" project, by Spanish Ministry of Science and Innovation, MICINN under Project MAT2010-18914 and by the Basque Government under Saiotek 11 MIMAGURA project (S- PE11UN087) and Saiotek-2011 MAGNANEL (S-E11UN085).

\section{CONFLICT OF INTEREST}

Declared none.

\section{REFERENCES}

[1] Jiles DC. Recent advances and future directions in magnetic materials. Acta Mater 2003; 51: 5907-39.

[2] Zhukova V, Ipatov M, Zhukov A. Thin magnetically soft wires for magnetic microsensors. Sensors 2009; 9: 9216-40.

[3] Cobeño AF, Zhukov A, Blanco JM, Gonzalez J. Giant magnetoimpedance effect in $\mathrm{CoMnSiB}$ amorphous microwires. J Magn Magn Mater 2001; 234: L359- 65.

[4] Óvári TA, Corodeanu S, Chiriac H. Domain wall velocity in submicron amorphous wires. J Appl Phys 2011; 109: 07D502.

[5] Honkura Y. Development of amorphous wire type MI sensors for automobile use. J Magn Magn Mater 2002; 249: 375-81.

[6] Panina LV, Mohri K. Magneto-impedance effect in amorphous wires. Appl Phys Lett 1994; 65: 1189-91.

[7] Beach R, Berkowitz A. Giant magnetic field dependent impedance of amorphous FeCoSiB wire. Appl Phys Lett 1994; 64: 3652-4.
[8] Zhukov AP. The remagnetization process of bistable amorphous alloys. Mater Des 1993; 5: 299.

[9] Shimode A, Hamada N, Mori M, Nagao T, Yamamoto M, Honkura Y. IWMW 2010 Proceedings. Bodrum: Turkey, p. 19.

[10] Uchiyama T, Mohri K, Nakayama S. Measurement of spontaneous oscillatory magnetic field of guinea-pig smooth muscle preparation using pico-tesla resolution amorphous wire magneto-impedance sensor. IEEE Trans Magn 2011; 47(10): 3070-3.

[11] Ipatov M, Zhukova V, Zhukov A, Gonzalez J, Zvezdin A. Lowfield hysteresis in the magnetoimpedance of amorphous microwires. Phys Rev B 2010; 81: 134421-8.

[12] Sandacci SI, Makhnovskiy DP, Panina LV, Mohri K, Honkura Y. Off-diagonal impedance in amorphous wires and its application to linear magnetic sensors. IEEE Trans Magn 2004; 35: 3505-10.

[13] Zhukov A, Zhukova V. Magnetic properties and applications of ferromagnetic microwires with amorphous and nanocrystalline structure. NY, USA: Nova Science Publishers 2009; p. 162.

[14] Zhukov A, Ipatov M, Blanco JM, Gonzalez J, Zhukova V. Studies of thin microwires with enhanced magnetic softness and GMI effect. Phys Stat Sol A 2009; 206(4), 674-8.

[15] Zhukova V, Usov NA, Zhukov A, Gonzalez J. Length effect in Corich amorphous wire. Phys Rev B 2002; 65:134407.

[16] Zhukov A, Ipatov M, Gonzalez J, Blanco JM, Zhukova V. Recent advances in studies of magnetically soft amorphous microwires. J Magn Magn Mater 2009; 321: 822-5.

[17] Fujimori H, Arai KI, Shirae H, Saito H, Masumoto T, Tsuya N. Magnetostriction of FeCo amorphous alloy. J Appl Phys 1976; 15: 705-6.

[18] Chiriac H, Ovari TA, Pop GH. Internal stress distribution in glasscovered amorphous magnetic wires. Phys Rev B 1995; 42:.1010513 .

[19] Zhukov A, Ipatov M, Zhukova V. Development of magnetically soft microwires with GMI effect. J Phys Conf Ser 2011; 303: 012085 .

[20] Zhukov A. Design of magnetic properties of Fe-rich glass - coated magnetic microwires for technical applications. Adv Funct Mater 2006; $16: 675-80$. 